\title{
Slepton mass-splittings as a signal of LFV at the LHC
}

\author{
Andrzej J. Buras, ${ }^{1,2}$ Lorenzo Calibbi,${ }^{3}$ and Paride Paradisi ${ }^{1}$ \\ ${ }^{1}$ Physik-Department, Technische Universität München, D-85748 Garching, Germany \\ ${ }^{2}$ TUM Institute for Advanced Study, Technische Universität München, \\ Arcisstr. 21, D-80333 München, Germany \\ ${ }^{3}$ Max-Planck-Institut für Physik (Werner-Heisenberg-Institut), D-80805 München, Germany
}

\begin{abstract}
Precise measurements of slepton mass-splittings might represent a powerful tool to probe supersymmetric (SUSY) lepton flavour violation (LFV) at the LHC. We point out that mass-splittings of the first two generations of sleptons are especially sensitive to LFV effects involving $\tau-\mu$ transitions. If these mass-splittings are LFV induced, high-energy LFV processes like the neutralino decay $\tilde{\chi}_{2}^{0} \rightarrow \tilde{\chi}_{1}^{0} \tau^{ \pm} \mu^{\mp}$ as well as low-energy LFV processes like $\tau \rightarrow \mu \gamma$ are unavoidable. We show that precise slepton mass-splitting measurements and LFV processes both at the high- and low-energy scales are highly complementary in the attempt to (partially) reconstruct the flavour sector of the SUSY model at work. The present study represents another proof of the synergy and interplay existing between the LHC, i.e. the high-energy frontier, and high-precision low-energy experiments, i.e. the high-intensity frontier.
\end{abstract}

\section{INTRODUCTION}

The most important achievement we expect to reach at the beginning of the LHC era is the understanding of the underlying mechanism accounting for the electroweak symmetry breaking, in particular, whether the Higgs mechanism is realized in nature or not. Moreover, the LHC is also expected to shed light on the hierarchy problem, since a natural solution of it calls for a $\mathrm{TeV}$ scale New Physics (NP).

On the other hand, low-energy flavour physics observables provide the most powerful tool to unveil the symmetry properties of the NP theory that will emerge at the LHC, if any. In fact, high-precision measurements at the LHC are made typically challenging by the huge background and by irreducible hadronic uncertainties.

A remarkable exception, arising in SUSY theories, is given by the possibility to access information about SUSY masses relying on some kinematical observables, as the kinematic end-point of the invariant mass distribution of the leptons coming from neutralino-sleptonneutralino cascade decays [1], $\tilde{\chi}_{2}^{0} \rightarrow \tilde{\ell}^{ \pm} \ell^{\mp} \rightarrow \tilde{\chi}_{1}^{0} \ell^{ \pm} \ell^{\mp}$. If the slepton in the decay chain is real, the di-lepton invariant mass spectrum has a prominent kinematic edge 1] that may be measured at the LHC experiments with very high precision (up to $0.1 \%$ ) [1, 2], allowing, in combination with other kinematical observables, to reconstruct the masses of the particles involved in the chain, in particular the slepton masses [1].

In this work, we point out that precise measurements of slepton mass-splittings might represent a powerful tool to probe LFV at the LHC.

In particular, we consider minimal gravity mediated SUSY breaking scenarios (mSUGRA), where the first two slepton generations are predicted to be highly degenerate, typically below the percent level. Hence, any experimental evidence for a sizable mass splitting between selectrons and smuons $\left(\Delta m_{\tilde{\ell}} / m_{\tilde{\ell}}\right)$, say above the percent level, would signal either a different mechanism for SUSY breaking or non minimal realizations of SUGRA breaking models. In the latter case, the presence of LFV interactions might be at the origin of such a significant mass splitting $\Delta m_{\tilde{\ell}} / m_{\tilde{\ell}}$.

In this context, we point out that sizable values for $\Delta m_{\tilde{\ell}} / m_{\tilde{\ell}}$ can be generated only through flavour mixings between the second and third slepton families, as it might naturally arise from the large mixing angle observed in atmospheric neutrino oscillation experiments. In contrast, flavour mixings between the second and first slepton families are tightly constrained by the non observation of $\mu \rightarrow e \gamma$, hence, they cannot induce testable values for $\Delta m_{\tilde{\ell}} / m_{\tilde{\ell}}$, unless very special and fine-tuned conditions are fulfilled [3].

If these mass-splittings are LFV induced, high-energy LFV processes like the neutralino decay $\tilde{\chi}_{2}^{0} \rightarrow \tilde{\chi}_{1}^{0} \tau^{ \pm} \mu^{\mp}$ [4 6] as well as low-energy LFV processes like $\tau \rightarrow \mu \gamma$ are unavoidable. We show that precise slepton masssplitting measurements and LFV processes (both at the high- and low-energy scales) are highly complementary in the attempt to (partially) reconstruct the flavour sector of the SUSY model at work.

Our paper is organized as follows. In Section II we summarize those slepton mass measurements at the LHC that are relevant for our paper. Subsequently in Section III we discuss various aspects of LFV at the LHC. In Section IV we present the numerical analysis of LFV and in Sections V] and VI estimates of the cross-sections, expected number of events at the LHC and backgrounds for the relevant processes discussed in our paper are made. In Section VII we summarize our main findings.

\section{SLEPTON MASS MEASUREMENTS AT THE LHC}

Sleptons can be produced at the LHC in two possible ways: either directly in quark collisions, through DrellYan s-channel $Z^{0} / \gamma$ exchange, or indirectly from cascade decays of squarks and gluinos through neutralinos. In the case of direct production, the detection of sleptons is 
made challenging by relatively low cross-section and the large SM background and it should be feasible only for slepton masses up to $200-300 \mathrm{GeV}$ 7]. Moreover, the indetermination of the center of mass energy of the parent quarks makes really difficult to extract information about the slepton masses. On the other hand, sleptons can be copiously produced in cascade decays of squarks through neutralinos, if the processes in the chain are kinematically allowed. Within several SUSY models, such as the CMSSM, where the second-lightest neutralino is mostly Wino-like, one of the most effective of such chains is

$$
\tilde{q}_{L} \rightarrow q_{L} \tilde{\chi}_{2}^{0} \rightarrow q_{L} \tilde{\ell}^{ \pm} \ell^{\mp},
$$

with typically $\operatorname{BR}\left(\tilde{q}_{L} \rightarrow q_{L} \tilde{\chi}_{2}^{0}\right) \simeq 1 / 3$. The slepton will typically decay into a lepton and the LSP. Besides the possibly large amount of sleptons produced in this way (clearly if $m_{\tilde{\ell}}<m_{\tilde{\chi}_{2}}$ ), the main advantage with respect to the direct Drell-Yan production is given by the possibility to access information about SUSY masses relying on some kinematical observables, which may be measured very precisely at the LHC.

An example of such observables, which is particularly important in the case of sleptons, is given by the kinematic end-point of the invariant mass distribution of the leptons coming from neutralino-slepton-neutralino cascade decays [1],

$$
\tilde{\chi}_{2}^{0} \rightarrow \tilde{\ell}^{ \pm} \ell^{\mp} \rightarrow \tilde{\chi}_{1}^{0} \ell^{ \pm} \ell^{\mp} .
$$

If the slepton in the decay chain is real, the di-lepton invariant mass spectrum has a prominent kinematic edge [1] at

$$
m_{l l}^{2}=\frac{\left(m_{\tilde{\chi}_{2}^{0}}^{2}-m_{\tilde{\ell}}^{2}\right)\left(m_{\tilde{\ell}}^{2}-m_{\tilde{\chi}_{1}^{0}}^{2}\right)}{m_{\tilde{\ell}}^{2}} .
$$

Such an edge may be measured at the LHC experiments with very high precision (up to $0.1 \%$ ) [1, 2], allowing, in combination with other kinematic observables, to reconstruct the masses of the particles involved in the chain, in particular the slepton masses [1] .

Interestingly, in the region of the parameter space where decays of neutralino in real sleptons are kinematically allowed, $\operatorname{BR}\left(\tilde{\chi}_{2}^{0} \rightarrow \tilde{\chi}_{1}^{0} \ell^{ \pm} \ell^{\mp}\right)$ is clearly enhanced with respect to the case of virtual intermediate sleptons. Noteworthy enough, in the CMSSM, such region of the parameter space is close to the region where $m_{\tilde{\tau}_{1}} \simeq m_{\tilde{\chi}_{1}^{0}}$ (where $\tilde{\tau}_{1}$ is the lightest stau) and the WMAP dark-matter (DM) constraints are naturally satisfied by an efficient $\tilde{\tau}_{1}$-LSP coannihilation [6].

For our purposes, it is important to understand whether the measurement of the kinematic edge of Eq. (3) in the case of $\mu-\mu$ and $e-e$ mass distributions can be used to resolve a (small) mass difference between the corresponding sleptons ( $\tilde{\mu}$ and $\tilde{e}$, in our case).

The fractional shift in the invariant mass edge in terms of the slepton mass splitting $\Delta m_{\tilde{\ell}} / m_{\tilde{\ell}}$ is given by

$$
\frac{\Delta m_{l l}}{m_{l l}}=\frac{\Delta m_{\tilde{\ell}}}{m_{\tilde{\ell}}}\left(\frac{m_{\tilde{\chi}_{1}^{0}}^{2} m_{\tilde{\chi}_{2}^{0}}^{2}-m_{\tilde{\ell}}^{4}}{\left(m_{\tilde{\chi}_{2}^{0}}^{2}-m_{\tilde{\ell}}^{2}\right)\left(m_{\tilde{\ell}}^{2}-m_{\tilde{\chi}_{1}^{0}}^{2}\right)}\right) \text {. }
$$

where the factor multiplying $\Delta m_{\tilde{\rho}} / m_{\tilde{\ell}}$ can provide an enhancement of the edge splitting [8].

The above equation deserves some comments: i) when $m_{\tilde{\ell}}=\sqrt{m_{\tilde{\chi}_{1}^{0}} m_{\tilde{\chi}_{2}^{0}}}$ the shift in the edge vanishes to leading order in $\Delta m_{\tilde{\ell}} / m_{\tilde{\ell}}$, ii) large splittings of the di-lepton edges can be achieved for relatively small splittings of the selectron and smuon masses and iii) the enhancement factor is larger for more degenerate masses of sparticles in the chain and can easily be $\mathcal{O}(10)$ depending upon the value of $m_{\tilde{\chi}_{2}^{0}} / m_{\tilde{\chi}_{1}^{0}}[\underline{8}]$. The enhancement diverges as the slepton mass approaches either neutralino masses. In the latter case, such an enhancement is not effective because the leptons coming from such chain tend to be soft and thus hard to detect and identify experimentally.

\section{A. Lepton flavour conserving case}

Within the CMSSM, the first two slepton generations are degenerate to a very large extent. In particular, in the absence of flavour mixing angles, the slepton masses $m_{\tilde{\ell}_{1,2}}$ are given by

$$
m_{\tilde{\ell}_{1,2}}^{2}=\frac{\left(m_{\tilde{\ell}_{L}}^{2}+m_{\tilde{\ell}_{R}}^{2}\right)}{2} \mp \frac{\sqrt{\left(m_{\tilde{\ell}_{L}}^{2}-m_{\tilde{\ell}_{R}}^{2}\right)^{2}+4\left(\Delta_{R L}^{\tilde{\ell} \tilde{\ell}}\right)^{2}}}{2}
$$

where $m_{\tilde{\ell}_{L(R)}}$ is the left-left (right-right) entry in the slepton mass matrix and $\Delta_{R L}^{\tilde{\ell} \tilde{\ell}}=m_{\ell}\left(A_{\ell}-\mu \tan \beta\right)$ is the leftright mixing term.

At leading order, $m_{\tilde{\ell}_{L}}^{2} \approx m_{0}^{2}\left(1-|c| y_{\ell}^{2}\right)+0.5 M_{1 / 2}^{2}$ and $m_{\tilde{\ell}_{R}}^{2} \approx m_{0}^{2}\left(1-2|c| y_{\ell}^{2}\right)+0.15 M_{1 / 2}^{2}$ where $m_{0}$ and $M_{1 / 2}$ are the universal soft sfermion and gaugino masses while $|c|$ stems from RGE effects driven by the Yukawa interactions and reads $|c| \approx\left(3+a_{0}^{2}\right) \ln \left(M_{X} / M_{Z}\right) /(4 \pi)^{2}$ (where $M_{X}$ is the high scale where the universality is imposed, i.e. either the Planck or the GUT scale and $a_{0} \equiv A_{0} / m_{0}$ is the universal trilinear coupling).

Since in the CMSSM $M_{\tilde{\chi}_{2}^{0}} \simeq 0.8 M_{1 / 2}$, the kinematic condition $m_{\tilde{\chi}_{2}^{0}}>m_{\tilde{\ell}}$ is satisfied when $m_{0} \lesssim 0.35 M_{1 / 2}$ in case of LH sleptons, $m_{0} \lesssim 0.7 M_{1 / 2}$ in case of $\mathrm{RH}$ sleptons. In this regime, it is straightforward to check that $\left(m_{\tilde{\ell}_{L}}^{2}-m_{\tilde{\ell}_{R}}^{2}\right) \gg \Delta_{R L}^{\tilde{\ell} \tilde{\ell}}$ and Eq. (5) leads to

$$
\frac{\Delta m_{\tilde{\ell}}}{m_{\tilde{\ell}}} \simeq \frac{m_{\tilde{e}_{R}}-m_{\tilde{\mu}_{R}}}{m_{\tilde{\ell}}}+\frac{\left(\Delta_{R L}^{\tilde{\mu} \tilde{\mu}}\right)^{2}}{m_{\tilde{\ell}}^{2}\left(m_{\tilde{\mu}_{L}}^{2}-m_{\tilde{\mu}_{R}}^{2}\right)}
$$

where we have defined $m_{\tilde{\ell}}=\left(m_{\tilde{e}_{R}}+m_{\tilde{\mu}_{R}}\right) / 2$. Hence, even for very large $\tan \beta$ values, the mass splitting between selectrons and smuons, $\Delta m_{\tilde{\ell}} / m_{\tilde{\ell}}$, can reach at most the per mill level. Therefore, it is commonly assumed that $m_{e e}$ and $m_{\mu \mu}$ edges occur at identical values in the CMSSM.

In principle, the enhancement factor for the edge splitting discussed in the above section could still bring the edge splittings at the percent level.

In practice, since in the CMSSM $\tilde{\tau}_{R}$ is driven light at large $\tan \beta$ and, thus, it dominates the $\tilde{\chi}_{2}^{0}$ decay 

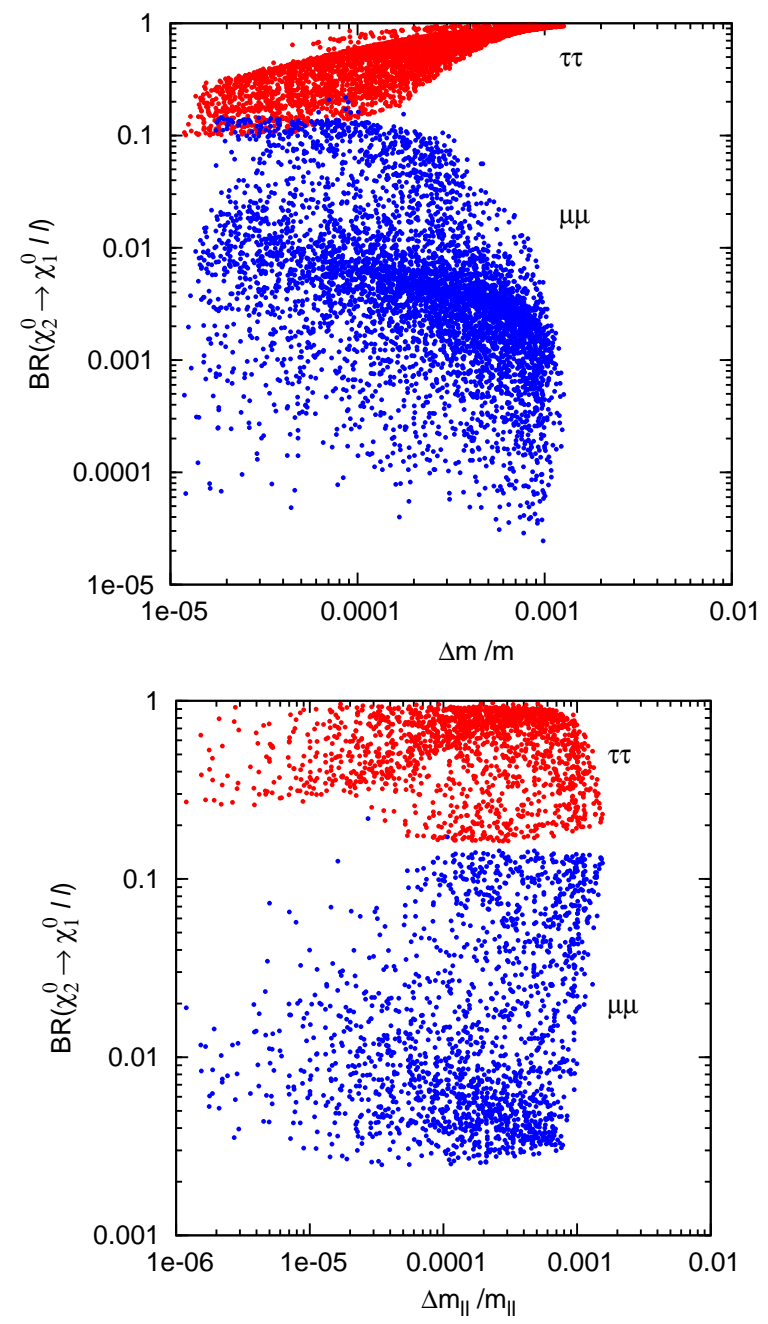

FIG. 1: $\operatorname{BR}\left(\tilde{\chi}_{2}^{0} \rightarrow \tilde{\chi}_{1}^{0} \ell \ell\right)$ (with $\left.\ell=\tau, \mu\right)$ vs. the slepton mass splitting $\Delta m_{\tilde{\ell}} / m_{\tilde{\ell}}$ (top) and the edge splitting $\Delta m_{l l} / m_{l l}$ (bottom).

modes, it turns out that $\operatorname{BR}\left(\tilde{\chi}_{2}^{0} \rightarrow \tilde{\tau}_{1} \tau\right) \sim 1$ while $\operatorname{BR}\left(\tilde{\chi}_{2}^{0} \rightarrow \tilde{\ell}_{R} \ell\right) \ll 1$ (with $\tilde{\ell}_{R}=\tilde{e}_{R}, \tilde{\mu}_{R}$ ), hence, the edge splitting turns out to be hardly measurable [8]. The above situation is well illustrated by Fig. 1 where, on the top, we show $\operatorname{BR}\left(\tilde{\chi}_{2}^{0} \rightarrow \tilde{\chi}_{1}^{0} \ell \ell\right.$ ) (with $\left.\ell=\tau, \mu\right)$ vs. the slepton mass splitting $\Delta m_{\tilde{\ell}} / m_{\tilde{\ell}}$ while on the bottom we show $\operatorname{BR}\left(\tilde{\chi}_{2}^{0} \rightarrow \tilde{\chi}_{1}^{0} \ell \ell\right)$ vs. the edge splitting $\Delta m_{l l} / m_{l l}$ (in this last case we impose the additional constraint of not too soft leptons, namely that $m_{\tilde{\chi}_{2}^{0}}-m_{\tilde{\ell}} \geq 10 \mathrm{GeV}$ and $m_{\tilde{\ell}}-m_{\tilde{\chi}_{1}^{0}} \geq 10 \mathrm{GeV}$ ). The plots of Fig. 1 have been obtained by means of a scan over the following ranges of the SUSY parameters: $m_{0}, M_{1 / 2} \leq 1 \mathrm{TeV}$, $-3 \leq a_{0} \leq+3$ and $\tan \beta \leq 50$. We see that for increasing values of $\Delta m_{\tilde{\ell}} / m_{\tilde{\ell}}, \operatorname{BR}\left(\tilde{\chi}_{2}^{0} \rightarrow \tilde{\chi}_{1}^{0} \tau \tau\right)$ increases as well while $\operatorname{BR}\left(\tilde{\chi}_{2}^{0} \rightarrow \tilde{\chi}_{1}^{0} \mu \mu\right)$ is suppressed. In any case, $\Delta m_{\tilde{\ell}} / m_{\tilde{\ell}}$ and $\Delta m_{l l} / m_{l l}$ are very small and they never exceed the per mill level.

We conclude that, any experimental evidence for a siz- able mass splitting between $\tilde{e}$ and $\tilde{\mu}$ would be a clear signal of a different mechanism for SUSY breaking than the CMSSM or non minimal realizations of SUGRA breaking models.

\section{B. Lepton flavour violating case}

As discussed before, within minimal SUGRA models, the first two slepton generations are highly degenerate. However, if we introduce LFV interactions, as it might be welcome in order to account for the neutrino masses and oscillations, a significant mass splitting $\Delta m_{\tilde{\ell}} / m_{\tilde{\ell}}$ can be still induced. In such a case, $\Delta m_{\tilde{\ell}} / m_{\tilde{\ell}}$ will turn out to be related to low energy LFV processes like $\ell_{i} \rightarrow \ell_{j} \gamma$.

We remind that, flavour mixings between the second and first slepton families are tightly constrained by the non observation of $\operatorname{BR}(\mu \rightarrow e \gamma)$, hence, they cannot typically induce testable values for $\Delta m_{\tilde{\ell}} / m_{\tilde{\ell}}$. Still, as widely stressed in the literature [3], such tight constraints might be evaded thanks to cancellations among different contributions to $\operatorname{BR}(\mu \rightarrow e \gamma)$ when the main source of LFV arises from the RH slepton sector. An example of such situation is studied in Ref. [3], in the context of a non-universal Higgs mass (NUHM) model. In that case, $\Delta m_{\tilde{\ell}} / m_{\tilde{\ell}} \sim 1 \%$ is still compatible with the present $\mu \rightarrow e \gamma$ bound, provided the SUSY input parameters are tuned to get exact cancellations in $\operatorname{BR}(\mu \rightarrow e \gamma)$.

On the other hand, we would like to point out that sizable values for $\Delta m_{\tilde{\ell}} / m_{\tilde{\ell}}$ can be more naturally generated through LFV in the $\tau-\mu$ (or $\tau-e$ ) sector, as it might naturally arise from the large mixing angle observed in atmospheric neutrino oscillation experiments.

In order to see this, let us consider for simplicity the illustrative case of a CMSSM with a single source of LFV that we parameterize, as usual, by means of the mass insertions (MIs) $\left(\delta_{\mathrm{XY}}\right)_{i j} \equiv\left(\tilde{m}_{\mathrm{XY}}^{2}\right)_{i j} / \sqrt{\left(\tilde{m}_{\mathrm{XY}}^{2}\right)_{i i}\left(\tilde{m}_{\mathrm{XY}}^{2}\right)_{j j}}$ where $i, j=1,3$ are flavour indices, $\mathrm{X}, \mathrm{Y}=\mathrm{L}, \mathrm{R}$ refers to the chirality of the corresponding SM fermions and $\left(\tilde{m}_{\mathrm{XY}}^{2}\right)_{i j}$ are the $3 \times 3$ blocks of the slepton mass matrix with given chirality XY.

In such a case, we obtain two mass eigenstates that are a mixture of staus and smuons such that $m_{\tilde{\ell}_{1,2}}^{2}=$ $m_{\tilde{\hat{\ell}}}^{2}\left(1 \mp \delta_{32}\right)$, where for the moment we are neglecting the effect of the LR stau mixing term.

Given that in the absence of LFV sources all the three slepton generations were degenerate, a mass splitting between the third and the second generations will clearly imply also a mass splitting between the first two slepton generations, $\Delta m_{\tilde{\ell}} / m_{\tilde{\ell}}$.

It is straightforward to check that, in terms of the mass insertion $\delta_{32}$, such mass splitting is approximately given by

$$
\left|\frac{\Delta m_{\tilde{\ell}}}{m_{\tilde{\ell}}}\right| \simeq \frac{\left|\delta_{32}\right|}{2}
$$

Hereafter, we denote both the mass splitting between 
$\tilde{e}_{L}-\tilde{\mu}_{L}$ and $\tilde{e}_{R}-\tilde{\mu}_{R}$ (induced by $\left(\delta_{\mathrm{LL}}\right)_{32}$ and $\left(\delta_{\mathrm{RR}}\right)_{32}$, respectively) with $\Delta m_{\tilde{\ell}}$.

The mass splitting of Eq. (7) might be measured by the LHC with an accuracy level better than the percent [8], hence, such a measurement would enable us to test the presence of LFV effects in some classes of SUSY theories, by detecting flavour conserving processes such as $\tilde{\chi}_{2}^{0} \rightarrow$ $\tilde{\chi}_{1}^{0} e^{+} e^{-}, \tilde{\chi}_{2}^{0} \rightarrow \tilde{\chi}_{1}^{0} \mu^{+} \mu^{-}$.

In passing, we notice that LFV sources in the $\tau-\mu$ sector clearly induce also a mass splitting between the third and second slepton generations. Therefore, in principle, one could make use of the edge splitting measurements for the $\tau-\tau$ and $\mu-\mu$ invariant mass distributions to extract information about the LFV source $\delta_{32}$. However, in practice, this could be hard because i) experimentally the edge measurement for the $\tau-\tau$ case is more challenging than the electron and muon ones, ii) the staus and the smuons are split even in the absence of LFV due to both RGE effects driven by the Yukawa interactions $\left(\propto y_{\tau}^{2}\right)$ and the presence of a left-right mixing term that affects mostly the stau masses. As a result, the measurement of the edge splitting for the $\mu-\mu$ and $e-e$ invariant mass distributions seems to be the most suitable tool to unveil LFV effects.

Yet, it should be stressed that a mass splitting between smuons and selectrons does not necessarily represent a clear probe of LFV effects. In fact, one could always envisage a situation where the lepton and slepton mass matrices are enough aligned [9], in order to avoid LFV effects, while the masses for different slepton generations are non degenerate. The measurement of the slepton mass splittings in this latter case have been studied in Ref. [10].

Hence, in order to test whether the slepton mass splittings come from LFV sources, it is crucial to have direct signals from LFV processes as we are going to discuss in the following section.

\section{LFV AT THE LHC AND AT LOW-ENERGY EXPERIMENTS}

As discussed in the previous section, LFV sources for the $\tau-\mu$ transition naturally induce a smuon/selectron splitting that could be detected by means of edge splittings for the $\mu-\mu$ and $e-e$ invariant mass distributions of the processes $\tilde{\chi}_{2}^{0} \rightarrow \tilde{\chi}_{1}^{0} \mu^{ \pm} \mu^{\mp}$ and $\tilde{\chi}_{2}^{0} \rightarrow \tilde{\chi}_{1}^{0} e^{ \pm} e^{\mp}$. Obviously, at the same time, also the LFV process $\tilde{\chi}_{2}^{0} \rightarrow$ $\tilde{\chi}_{1}^{0} \tau^{ \pm} \mu^{\mp}$, that represents one of the most promising LFV decay mode at the LHC [5, 6, 11, 12], is generated.

In fact, the decay of $\tilde{\chi}_{2}^{0}$ in a lepton and slepton, followed by the decay of the slepton in a different lepton and the $\operatorname{LSP}\left(\operatorname{BR}\left(\tilde{\chi}_{2}^{0} \rightarrow \ell_{i} \ell_{j} \tilde{\chi}_{1}^{0}\right)\right)$, is a tree-level process, which simply depends on the misalignment between the slepton and lepton mass eigenstates [4]. Furthermore, there is no 'GIM-like' suppression $\left(\sim \Delta m_{\tilde{\ell}} / \bar{m}_{\tilde{\ell}}\right.$ in the amplitude) in the case of the intermediate sleptons being real $\left(m_{\tilde{\chi}_{2}^{0}}>m_{\tilde{\ell}_{\alpha}}\right)$.
Unfortunately, the broad parameter space in the CMSSM which can be probed by the LHC is already excluded by the $\mu \rightarrow e \gamma$ constraint when there is a LFV sources in the $\mu-e$ sector [3] (barring fine tuned cases where accidental cancellations strongly reduce $\operatorname{BR}(\mu \rightarrow$ er) [3] ).

Therefore, in the following, as mentioned above, we consider a possible mixing between $\tau$ - and $\mu$ sleptons (both left-handed and right-handed), since the constraints for LFV in the $\tau-\mu$ sector are less stringent than in the $\mu-e$ sector.

In principle, we could also consider the case of $\tilde{\tau}-\tilde{e}$ mixing. However, the possibility of having simultaneously large effects in $\tilde{\chi}_{2}^{0} \rightarrow \tilde{\chi}_{1}^{0} \tau^{ \pm} \mu^{\mp}$ and $\tilde{\chi}_{2}^{0} \rightarrow \tilde{\chi}_{1}^{0} \tau^{ \pm} e^{\mp}$, implying an effective $\mu-e$ mixing $\delta_{\mu e} \sim \delta_{\mu \tau} \delta_{\tau e}$, is again strongly constrained by the bounds on $\operatorname{BR}(\mu \rightarrow$ $e \gamma)$ 13, 14].

Therefore, neglecting the contribution of virtual intermediate sleptons, the branching ratio of the LFV neutralino decay can be written as:

$$
\begin{aligned}
\operatorname{BR}\left(\tilde{\chi}_{2}^{0} \rightarrow \ell_{i} \ell_{j} \tilde{\chi}_{1}^{0}\right)= & {\left[\operatorname{BR}\left(\tilde{\chi}_{2}^{0} \rightarrow \ell_{i} \tilde{\ell}_{\alpha}\right) \operatorname{BR}\left(\tilde{\ell}_{\alpha} \rightarrow \ell_{j} \tilde{\chi}_{1}^{0}\right)+\right.} \\
& \left.\operatorname{BR}\left(\tilde{\chi}_{2}^{0} \rightarrow \ell_{j} \tilde{\ell}_{\alpha}\right) \operatorname{BR}\left(\tilde{\ell}_{\alpha} \rightarrow \ell_{i} \tilde{\chi}_{1}^{0}\right)\right]
\end{aligned}
$$

where the sum is understood over the sleptons lighter than the neutralino. The relevant decay widths are given by [15]:

$$
\begin{aligned}
& \Gamma\left(\tilde{\chi}_{K}^{0} \rightarrow \tilde{\ell}_{\alpha} \ell_{i}\right)=\frac{\alpha_{2}}{16} m_{\tilde{\chi}_{K}^{0}}\left(1-\frac{m_{\tilde{\ell}_{\alpha}}^{2}}{m_{\tilde{\chi}_{K}^{0}}^{2}}\right)^{2}\left(\left|L_{i \alpha}^{K}\right|^{2}+\left|R_{i \alpha}^{K}\right|^{2}\right) \\
& \Gamma\left(\tilde{\ell}_{\alpha} \rightarrow \tilde{\chi}_{K}^{0} \ell_{i}\right)=\frac{\alpha_{2}}{8} m_{\tilde{\ell}_{\alpha}}\left(1-\frac{m_{\tilde{\chi}_{K}^{0}}^{2}}{m_{\tilde{\ell}_{\alpha}}^{2}}\right)^{2}\left(\left|L_{i \alpha}^{K}\right|^{2}+\left|R_{i \alpha}^{K}\right|^{2}\right)
\end{aligned}
$$

where the masses of ordinary leptons have been neglected, $K=1,4, \alpha=1,6$ and $i=1,3\left(l_{i}=(e, \mu, \tau)\right)$ and $L_{K \alpha}^{i}, R_{K \alpha}^{i}$ represent the lepton-slepton-neutralino interaction vertices:

$$
\begin{aligned}
& L_{i \alpha}^{K}=-\left[N_{K 2}+N_{K 1} \tan \theta_{W}\right] U_{\alpha i}+\frac{m_{l_{i}}}{M_{W} \cos \beta} N_{K 3} U_{\alpha(i+3)} \\
& R_{i \alpha}^{K}=2 N_{K 1} U_{\alpha(i+3)} \tan \theta_{W}+\frac{m_{l_{i}}}{M_{W} \cos \beta} N_{K 3} U_{\alpha i} .
\end{aligned}
$$

Here $U$ and $N$ are the matrices which rotate sleptons and neutralinos into their mass eigenstates.

In order to compute the total $\tilde{\chi}_{2}^{0}$ width, and then $\operatorname{BR}\left(\tilde{\chi}_{2}^{0} \rightarrow \tilde{\ell}_{\alpha} \ell_{j} \rightarrow \tilde{\chi}_{1}^{0} \ell_{i}^{ \pm} \ell_{j}^{\mp}\right)$, one has to consider the following flavour-violating and -conserving $\tilde{\chi}_{2}^{0}$ decays:

$$
\begin{aligned}
& \tilde{\chi}_{2}^{0} \rightarrow \tilde{\ell}_{\alpha} \ell_{j} \rightarrow \tilde{\chi}_{1}^{0} \ell_{i}^{ \pm} \ell_{j}^{\mp} \\
& \tilde{\chi}_{2}^{0} \rightarrow \tilde{\chi}_{1}^{0} Z^{0} \rightarrow \tilde{\chi}_{1}^{0} \ell_{i}^{+} \ell_{i}^{-} \\
& \tilde{\chi}_{2}^{0} \rightarrow \tilde{\chi}_{1}^{0} h^{0} \rightarrow \tilde{\chi}_{1}^{0} \ell_{i}^{+} \ell_{i}^{-} \\
& \tilde{\chi}_{2}^{0} \rightarrow \tilde{\nu}_{\alpha} \nu_{j} \rightarrow \tilde{\chi}_{1}^{0} \nu_{i} \nu_{j}
\end{aligned}
$$




\begin{tabular}{|l|c|c|r|}
\hline Process & Present Bound & Future Bound & Future Exp. \\
\hline \hline $\mathrm{BR}(\tau \rightarrow \mu \gamma)$ & $4.4 \times 10^{-8}$ & $\mathcal{O}\left(10^{-8}\right)$ & SuperB [17] \\
$\mathrm{BR}(\tau \rightarrow \mu \mu \mu)$ & $3.2 \times 10^{-8}$ & $\mathcal{O}\left(10^{-8}\right)$ & LHCb [18] \\
$\operatorname{BR}(\tau \rightarrow \mu e e)$ & $2.0 \times 10^{-8}$ & $\mathcal{O}\left(10^{-8}\right)$ & SuperB [17] \\
\hline
\end{tabular}

TABLE I: Present [16] and upcoming experimental limits on various $\tau-\mu$ transitions at 90\% C.L.

Clearly, the $h^{0}$ decays may provide a sizable rate only to the flavour-conserving decay $\tilde{\chi}_{2}^{0} \rightarrow \tilde{\chi}_{1}^{0} \tau_{i}^{ \pm} \tau_{i}^{\mp}$. Decays into squark-quark are not taken into account, since $\tilde{\chi}_{2}^{0}$ is always lighter than squarks in the SUSY framework considered here.

The presence of $\tau-\mu$ LFV sources will also induce, at one loop, LFV low energy processes like $\tau \rightarrow \mu \gamma$ and so on. In table I we report the current and expected experimental bounds on some of the $\tau-\mu$ transitions.

The branching ratio of $\tau \rightarrow \mu \gamma$ can be written as

$$
\frac{\operatorname{BR}(\tau \rightarrow \mu \gamma)}{\operatorname{BR}\left(\tau \rightarrow \mu \nu_{\tau} \overline{\nu_{\mu}}\right)}=\frac{48 \pi^{3} \alpha}{G_{F}^{2}}\left(\left|A_{L}^{32}\right|^{2}+\left|A_{R}^{32}\right|^{2}\right) .
$$

Although in the numerical analysis we perform a full computation of LFV processes using the exact formulae of Ref. [19], we report, in the following, the amplitudes as obtained in the MI approximation, within the illustrative case of a degenerate SUSY spectrum with a common mass $\tilde{m}[13]$ :

$$
\begin{aligned}
A_{L}^{32} & \simeq \frac{\alpha_{2}}{60 \pi} \frac{\tan \beta}{\tilde{m}^{2}}\left(\delta_{\mathrm{LL}}\right)_{32}, \\
A_{R}^{32} & \simeq-\frac{\alpha_{1}}{4 \pi} \frac{\tan \beta}{\tilde{m}^{2}} \frac{\left(\delta_{\mathrm{RR}}\right)_{32}}{60} .
\end{aligned}
$$

As we will discuss in detail in the next sections, $\tau \rightarrow$ $\mu \gamma$ and $\tilde{\chi}_{2}^{0} \rightarrow \tilde{\chi}_{1}^{0} \tau^{ \pm} \mu^{\mp}$ provide complementary probes of the SUSY parameter space, especially when combined also with the possible measurement of the edge splittings. First, we notice that $\operatorname{BR}(\tau \rightarrow \mu \gamma)$ scales as $\tan ^{2} \beta$ while, $\operatorname{BR}\left(\tilde{\chi}_{2}^{0} \rightarrow \tilde{\chi}_{1}^{0} \tau^{ \pm} \mu^{\mp}\right)$ is sensitive to $\tan \beta$ for kinematical reasons, even though it does not depend explicitly on it. Second, $\operatorname{BR}(\tau \rightarrow \mu \gamma) \sim \tilde{m}^{-4}$ and thus it decouples fast with the SUSY scale, in contrast to $\operatorname{BR}\left(\tilde{\chi}_{2}^{0} \rightarrow \tilde{\chi}_{1}^{0} \tau^{ \pm} \mu^{\mp}\right)$.

\section{NUMERICAL ANALYSIS}

In this section, we present our numerical results, obtained in the framework of the CMSSM in presence of LFV sources. In order to compute the SUSY spectrum, we numerically solved the full 1-loop RGEs of the MSSM, switching on LFV mass-insertions at low-energy. Then, we compute the relevant processes by means of a full calculation in the mass eigenstate basis. For each point of the parameter space, we impose the following constraints: (i) successful EWSB and absence of tachyonic particles; (ii) limits on SUSY masses from direct searches, (iii) all the currently available hadronic flavour constraints [20].
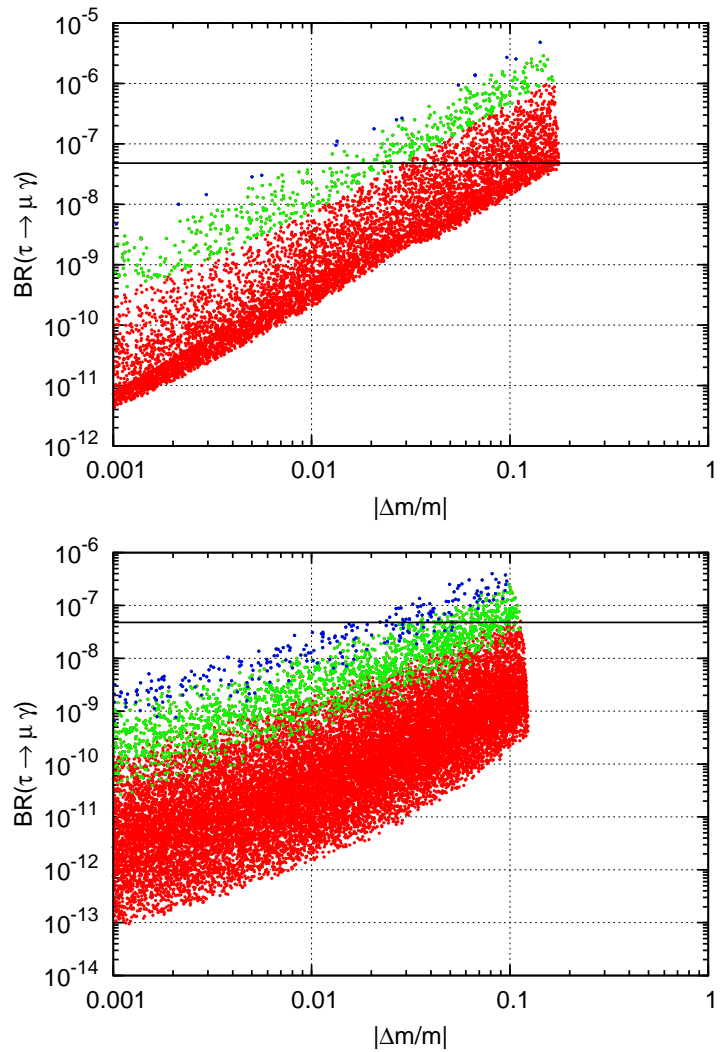

FIG. 2: Top: $\operatorname{BR}(\tau \rightarrow \mu \gamma)$ vs. $\Delta m_{\tilde{\ell}} / m_{\tilde{\ell}}$ in case of LFV sources in the left-left slepton sector. Bottom: the same for the right-right slepton sector.

In Fig. 2, we plot $\operatorname{BR}(\tau \rightarrow \mu \gamma)$ as a function of $\Delta m_{\tilde{\ell}} / m_{\tilde{\ell}}$, for the following choice of the SUSY parameters: $\tan \beta=10,0<m_{0}<1000 \mathrm{GeV}, 0<M_{1 / 2}<1000$ $\mathrm{GeV}, A_{0}=0$. In the upper plot, we switch on only the LFV MI $\left(\delta_{\mathrm{LL}}\right)_{32}$ and we vary it in the range $10^{-3}<$ $\left(\delta_{\mathrm{LL}}\right)_{32}<0.3$. All the points of Fig. 2 are such that the processes $\tilde{\chi}_{2}^{0} \rightarrow \tilde{\chi}_{1}^{0} e^{+} e^{-}$and $\tilde{\chi}_{2}^{0} \rightarrow \tilde{\chi}_{1}^{0} \mu^{+} \mu^{-}$through real sleptons are kinematically allowed, namely they satisfy $m_{\tilde{e}_{L}}, m_{\tilde{\mu}_{L}}<m_{\tilde{\chi}_{2}^{0}}$. In the lower plot of Fig. 2, we scan $\left(\delta_{\mathrm{RR}}\right)_{32}$ also in the range $10^{-3}<\left(\delta_{\mathrm{RR}}\right)_{32}<0.3$ and we require that $m_{\tilde{e}_{R}}, m_{\tilde{\mu}_{R}}<m_{\tilde{\chi}_{2}^{0}}$.

The grey points give $a_{\mu}^{\mathrm{SUSY}} \equiv(g-2)_{\mu}^{\mathrm{SUSY}} / 2>10^{-9}$, while for the blue points $a_{\mu}^{\text {SUSY }}>2 \times 10^{-9}$. The black horizontal line represents the present bound $\operatorname{BR}(\tau \rightarrow$ $\mu \gamma)<4.4 \times 10^{-8}$.

We observe that, in the case of left-handed sleptons, mass splittings of order $\lesssim 3 \%$, are compatible with a solution of the $(g-2)_{\mu}$ anomaly at the $2-\sigma$ level, i.e. $a_{\mu}^{\text {SUSY }}>10^{-9}$, while satisfying at the same time the $\tau \rightarrow \mu \gamma$ bound. Moreover, in the case of right-handed sleptons, the mass splitting can reach even the $10 \%$ level.

We have also found that, in the same ranges for the SUSY parameters as in Fig. 2, the edge splitting $\Delta m_{l l} / m_{l l}$ is unambiguously enhanced (at least by a factor of $\sim 3$ ) with respect to the slepton mass splitting $\Delta m_{\tilde{\ell}} / m_{\tilde{\ell}}$ when the LFV arises in the LL sector. In con- 

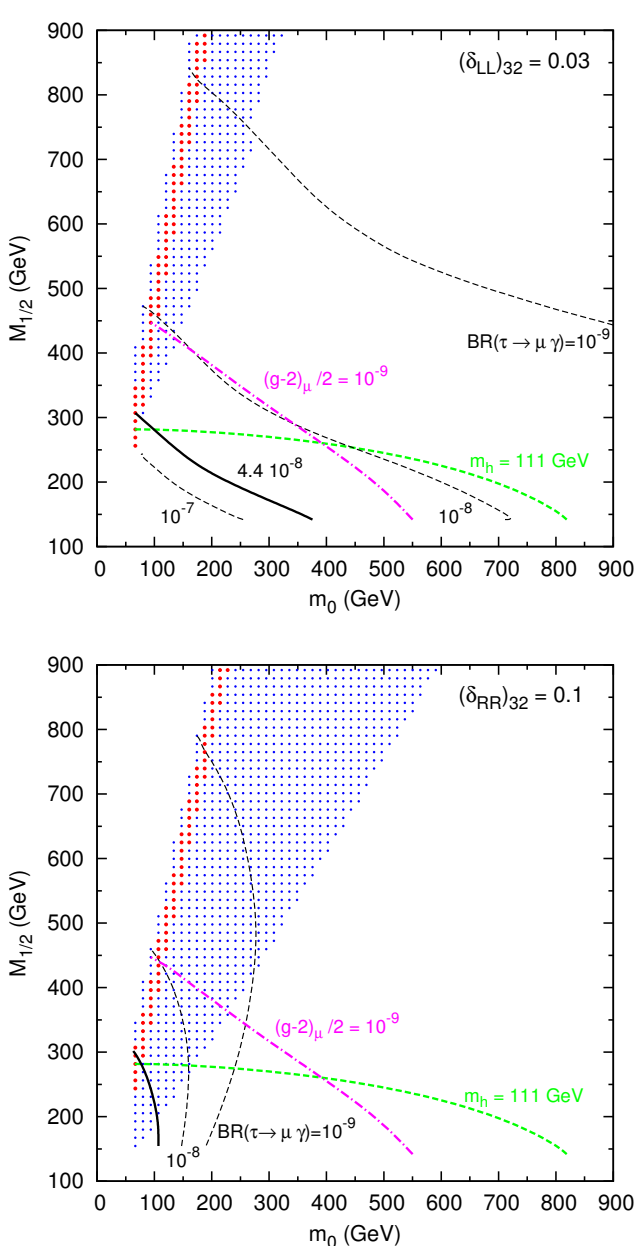

FIG. 3: Predictions for $\operatorname{BR}(\tau \rightarrow \mu \gamma)$ in the $\left(m_{0}, M_{1 / 2}\right)$ plane for $\tan \beta=10, A_{0}=0,\left(\delta_{\mathrm{LL}}\right)_{32}=0.03$ (top) and $\left(\delta_{\mathrm{RR}}\right)_{32}=0.1$ (bottom). In both plots, the process $\tilde{\chi}_{2}^{0} \rightarrow \tilde{\chi}_{1}^{0} \ell \ell$ (mediated by a real slepton) is kinematically allowed in the region marked with blue dots. The red points additionally satisfy the Dark Matter constraints.

trast, for LFV sources in the RR sector, $\Delta m_{l l} / m_{l l}$ can be both enhanced or suppressed (up to a factor of $\sim 3$ ) compared to $\Delta m_{\tilde{\ell}} / m_{\tilde{\ell}}$.

Since a precise measurement of the slepton masses would require $m_{\tilde{\ell}}<m_{\tilde{\chi}_{2}^{2}}$, it is useful to display the values of $m_{0}$ and $M_{1 / 2}$ accounting for $m_{\tilde{\ell}}<m_{\tilde{\chi}_{2}^{0}}$.

In Fig. 3, we show the $\left(m_{0}, M_{1 / 2}\right)$ plane for $\tan \beta=$ $10, A_{0}=0,\left(\delta_{\mathrm{LL}}\right)_{32}=0.03$ (top) and $\left(\delta_{\mathrm{RR}}\right)_{32}=0.1$ (bottom). We chose the above values of the LFV sources as illustrative cases of scenarios where $\operatorname{BR}(\tau \rightarrow \mu \gamma)$ is kept under control and, at the same time, sizable SUSY contributions to $(g-2)_{\mu}$ are still possible, as shown by Fig. 2 ,

The region of Fig. 3 marked with blue dots corresponds to the kinematically allowed region for the decays of neutralinos into smuons and selectrons. Their branching fractions here are always above the percent level $(\gtrsim 3 \%)$ in the case of $\left(\delta_{\mathrm{LL}}\right)_{32}=0.03$, while, for $\left(\delta_{\mathrm{RR}}\right)_{32}=0.1$,
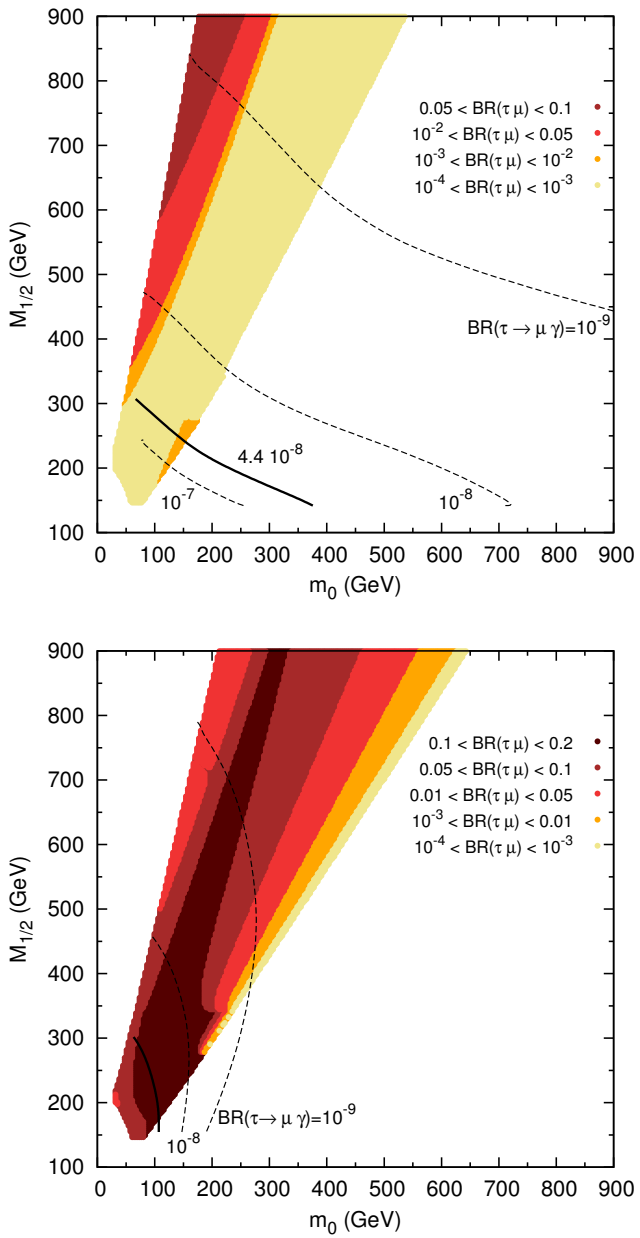

FIG. 4: Predictions for $\operatorname{BR}\left(\tilde{\chi}_{2}^{0} \rightarrow \tilde{\chi}_{1}^{0} \tau^{ \pm} \mu^{\mp}\right)$ and $\operatorname{BR}(\tau \rightarrow$ $\mu \gamma)$ in the $\left(m_{0}, M_{1 / 2}\right)$ plane for $\tan \beta=10, A_{0}=0$, $\left(\delta_{\mathrm{LL}}\right)_{32}=0.03$ (top) and $\left(\delta_{\mathrm{RR}}\right)_{32}=0.1$ (bottom). The $\operatorname{BR}\left(\tilde{\chi}_{2}^{0} \rightarrow \tilde{\chi}_{1}^{0} \tau^{ \pm} \mu^{\mp}\right)$ decreases passing from the darker to the lighter regions.

they are around $1 \%$ in the region favoured by $(g-2)_{\mu}$ and get lower outside it. This region includes also the neutralino-stau coannihilation strip (red dots).

In the upper plot, the MI $\left(\delta_{\mathrm{LL}}\right)_{32}=0.03$ induces a mass splitting $\left(\Delta m_{\tilde{\ell}} / m_{\tilde{\ell}}\right)_{L}$ around 1-1.5\%. In the lower plot, the MI $\left(\delta_{\mathrm{RR}}\right)_{32}=0.1$ gives $2 \% \lesssim\left(\Delta m_{\tilde{\ell}} / m_{\tilde{\ell}}\right)_{R} \lesssim 4 \%$. In both figures, the black thick line refers to the current bound on $\operatorname{BR}(\tau \rightarrow \mu \gamma)$, the purple dashed-dot line corresponds to $a_{\mu}^{\mathrm{SUSY}}=1 \times 10^{-9}$ while the green dashed line accounts for the lightest Higgs boson bound (we impose $m_{h}>111 \mathrm{GeV}$ taking into account a theoretical uncertainty of $3 \mathrm{GeV}$ ).

Interestingly enough, both plots of Fig. 3 show that there are sizable regions of the parameter space where, at the same time: (i) $\operatorname{BR}(\tau \rightarrow \mu \gamma)$ satisfies the current experimental bound while being within the reach of a SuperB factory at $\operatorname{KEK}\left(\operatorname{BR}(\tau \rightarrow \mu \gamma)>10^{-8}[17]\right)$, (ii) the $(g-2)_{\mu}$ anomaly can be explained at the $2-\sigma$ level, i.e. $a_{\mu}^{\mathrm{SUSY}} \gtrsim 1 \times 10^{-9}$; (iii) the WMAP relic den- 

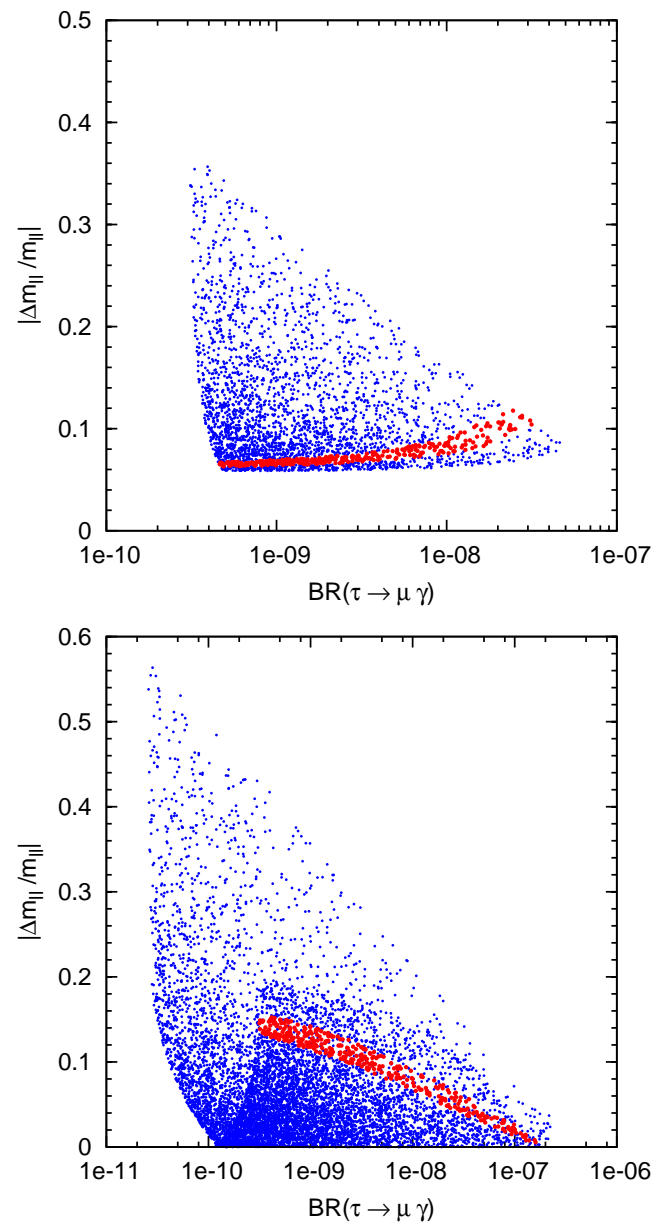

FIG. 5: $\left|\Delta m_{l l} / m_{l l}\right|$ vs. $\operatorname{BR}(\tau \rightarrow \mu \gamma)$ for the points corresponding to the blue and red regions of Fig. 3

sity constraint can be fulfilled by an effective neutralinostau coannihilation; (iv) selectrons and smuons can be produced by cascade decays through the next-to-lightest neutralino.

In Fig. 4 we show the predictions for $\operatorname{BR}\left(\tilde{\chi}_{2}^{0} \rightarrow\right.$ $\left.\tilde{\chi}_{1}^{0} \tau^{ \pm} \mu^{\mp}\right)$ and $\operatorname{BR}(\tau \rightarrow \mu \gamma)$ in the $\left(m_{0}, M_{1 / 2}\right)$ plane for the same input parameters as in Fig. 3. We notice that the kinematically allowed region of Fig. 4 is significantly larger than that of Fig. 3. The reason can be traced back remembering that the staus are lighter than $\tilde{\chi}_{2}^{0}$ in a broader region of the SUSY parameter space compared to the selectrons and smuons. In these plots, $\operatorname{BR}\left(\tilde{\chi}_{2}^{0} \rightarrow \tilde{\chi}_{1}^{0} \tau^{ \pm} \mu^{\mp}\right)$ decreases passing from the darker to the lighter regions.

Interestingly enough, Fig. 4 clearly shows that $\operatorname{BR}\left(\tilde{\chi}_{2}^{0} \rightarrow \tilde{\chi}_{1}^{0} \tau^{ \pm} \mu^{\mp}\right)$ and $\operatorname{BR}(\tau \rightarrow \mu \gamma)$ are complementary probes of LFV in SUSY with $\operatorname{BR}\left(\tilde{\chi}_{2}^{0} \rightarrow \tilde{\chi}_{1}^{0} \tau^{ \pm} \mu^{\mp}\right)$ being potentially more powerful than $\operatorname{BR}(\tau \rightarrow \mu \gamma)$ especially for a heavy SUSY spectrum.

As mentioned above, a smuon/selectron mass splitting at the percent level would be potentially measurable at the LHC especially if the edge splitting $\Delta m_{l l} / m_{l l}$ receives an enhancement. In order to check this crucial point, in
Fig. 5 we plot $\left|\Delta m_{l l} / m_{l l}\right|$ (as generated by LFV effects) vs. $\operatorname{BR}(\tau \rightarrow \mu \gamma)$, selecting the points corresponding to the blue-dotted region of Fig. 3. We impose on those points the additional requirements $\left(m_{\tilde{\chi}_{2}^{0}}-m_{\tilde{e}, \tilde{\mu}}\right) \geq 10$ $\mathrm{GeV},\left(m_{\tilde{e}, \tilde{\mu}}-m_{\tilde{\chi}_{1}^{0}}\right) \geq 10 \mathrm{GeV}$, so that the leptons are not too soft for detection.

As shown by Fig. $5 . \Delta m_{l l} / m_{l l}$ might be quite large, well beyond the $10 \%$ level, even for $\operatorname{BR}(\tau \rightarrow \mu \gamma) \lesssim 10^{-9}$ thus beyond the reach of a Super Flavour Factory [21].

Focusing on the coannihilation strip, corresponding to the red points of Fig. 5, we observe that, in the case of $\left(\delta_{\mathrm{LL}}\right)_{32}=0.03$, the splitting of the $\mu-\mu$ and $e-e$ edges (coming from cascade decays through $\tilde{\mu}_{L}$ and $\tilde{e}_{L}$, respectively) is around $8 \%$. For $\left(\delta_{\mathrm{RR}}\right)_{32}=0.1$, the edge splitting (now corresponding to intermediate $\tilde{\mu}_{R}$ and $\tilde{e}_{R}$ ) can be of order $10 \%$ or more.

The above edge splittings, that are well measurable at the LHC, can probe the SUSY parameter space in regions where $\operatorname{BR}(\tau \rightarrow \mu \gamma)$ is experimentally challenging or even unreachable.

\section{PRODUCTION CROSS-SECTIONS, SIGNALS AND BACKGROUNDS}

In the following, we are going to estimate the crosssections, signals and backgrounds for the relevant processes discussed in the previous sections, in order to provide information about the capability of the LHC of detecting such processes and, in particular, of measuring the slepton masses thanks to the kinematical end-points technique.

A full simulation of the production, decays and detection of SUSY particles at the LHC is beyond the scope of the present paper. Nevertheless, we can get a semiquantitative idea of the size of the cross-sections, signals and backgrounds, by using some approximate formulae provided in the literature.

\section{A. Production cross-sections}

At the LHC, operating at $14 \mathrm{TeV}$, the total production cross-section for squarks and gluinos, within the CMSSM, can be estimated to be [5]:

$$
\sigma_{\mathrm{SUSY}} \simeq 1.79 \times 10^{13}\left(0.1 m_{0}+M_{1 / 2}\right)^{-4.8} \mathrm{pb} .
$$

This formula gives an agreement with the full computation to within $25 \%[3,5]$.

In order to estimate the number of (Wino-like) $\tilde{\chi}_{2}^{0}$ produced from cascade of squarks and gluinos, we will take $\operatorname{BR}\left(\tilde{q}_{L} \rightarrow q_{L} \tilde{\chi}_{2}^{0}\right) \simeq 1 / 3$ and assume the probability to produce a $\tilde{q}_{L}$ to be $50 \%$. Hence, the production crosssection of $\tilde{\chi}_{2}^{0}$ can be estimated to be:

$$
\sigma_{\tilde{\chi}_{2}^{0}} \simeq \frac{1}{2} \sigma_{\mathrm{SUSY}} \times \operatorname{BR}\left(\tilde{q}_{L} \rightarrow q_{L} \tilde{\chi}_{2}^{0}\right) \simeq \frac{1}{6} \sigma_{\mathrm{SUSY}} .
$$


Making extensive use of the formulae given above and computing numerically the branching fractions of the second neutralino decays, we are going to estimate the following quantities:

$$
\begin{aligned}
\sigma_{e e} & \equiv \sigma\left(\tilde{\chi}_{2}^{0} \rightarrow \tilde{\chi}_{1}^{0} e^{+} e^{-}\right)=\sigma_{\tilde{\chi}_{2}^{0}} \times \operatorname{BR}\left(\tilde{\chi}_{2}^{0} \rightarrow \tilde{\chi}_{1}^{0} e^{+} e^{-}\right) \\
\sigma_{\mu \mu} & \equiv \sigma\left(\tilde{\chi}_{2}^{0} \rightarrow \tilde{\chi}_{1}^{0} \mu^{+} \mu^{-}\right)=\sigma_{\tilde{\chi}_{2}^{0}} \times \operatorname{BR}\left(\tilde{\chi}_{2}^{0} \rightarrow \tilde{\chi}_{1}^{0} \mu^{+} \mu^{-}\right) \\
\sigma_{\tau \mu} & \equiv \sigma\left(\tilde{\chi}_{2}^{0} \rightarrow \tilde{\chi}_{1}^{0} \tau^{ \pm} \mu^{\mp}\right)=\sigma_{\tilde{\chi}_{2}^{0}} \times \operatorname{BR}\left(\tilde{\chi}_{2}^{0} \rightarrow \tilde{\chi}_{1}^{0} \tau^{ \pm} \mu^{\mp}\right)
\end{aligned}
$$

\section{B. Signals and backgrounds}

Let us now estimate signals and relevant backgrounds following closely the analysis of Ref. [11]). We are considering opposite sign dilepton events (flavour conserving and flavour violating ones), which can have both SM and SUSY backgrounds.

The SM background mainly comes from $W^{+} W^{-}$and $t \bar{t}$ production. Both these sources can be strongly reduced by imposing the standard cuts (on missing $p_{T}$, number of jets, jets $p_{T}$, etc.) used to discriminate SUSY events from the SM background (see for instance the discussion in Ref. [5, 6]). We will therefore concentrate only on the SUSY background assuming that the SM one can be sufficiently reduced.

We will parameterize the effect of the above mentioned kinematic cuts by means of an acceptance factor $\epsilon_{\text {cut }}$, affecting both our signals and the corresponding SUSY background.

Let us start considering the opposite sign same flavour events we want to study for extracting the slepton masses. The signal is given by:

$$
S_{\ell^{+} \ell^{-}}=\sigma_{\ell \ell} \times \epsilon_{\ell}^{2} \times \epsilon_{\mathrm{cut}} \times L,
$$

where $\epsilon_{\ell}$ is the lepton detection efficiency and $L$ the integrated luminosity.

There are two main SUSY sources of background to these events. The first one comes from pairs of lefthanded squark-antisquark, when both the particles decay into opposite sign charginos:

$$
\tilde{q}_{L} \tilde{q}_{L}^{*} \rightarrow \tilde{\chi}_{1}^{+} \tilde{\chi}_{1}^{-}+\cdots
$$

The charginos can then produce opposite sign leptons (either with same or different flavours) by decaying as follows:

$$
\begin{aligned}
& \tilde{\chi}_{1}^{ \pm} \rightarrow \tilde{\nu} \ell^{ \pm}, \\
& \tilde{\chi}_{1}^{ \pm} \rightarrow \tilde{\ell}^{ \pm} \nu \rightarrow \ell^{ \pm} \nu \tilde{\chi}^{0}, \\
& \tilde{\chi}_{1}^{ \pm} \rightarrow W^{ \pm} \tilde{\chi}^{0} \rightarrow \ell^{ \pm} \nu \tilde{\chi}^{0} .
\end{aligned}
$$

The number of such background events can be estimated to be:

$$
\begin{aligned}
B_{\ell^{+} \ell^{-}}^{\tilde{\chi}^{+} \tilde{\chi}^{-}} & =\sigma_{\tilde{\chi}^{+} \tilde{\chi}^{-}} \times \epsilon_{\ell}^{2} \times \epsilon_{\mathrm{cut}} \times L \times \\
& \times\left[\operatorname{BR}\left(\tilde{\chi}_{1}^{ \pm} \rightarrow \tilde{\nu} \ell^{ \pm}\right)+\right. \\
& +\operatorname{BR}\left(\tilde{\chi}_{1}^{ \pm} \rightarrow \tilde{\ell}^{ \pm} \nu\right) \operatorname{BR}\left(\tilde{\ell}^{ \pm} \rightarrow \ell^{ \pm} \tilde{\chi}^{0}\right)+ \\
& \left.+\operatorname{BR}\left(\tilde{\chi}_{1}^{ \pm} \rightarrow W^{ \pm} \tilde{\chi}^{0}\right) \operatorname{BR}\left(W^{ \pm} \rightarrow \ell^{ \pm} \nu\right)\right]^{2}
\end{aligned}
$$

where $\sigma_{\tilde{\chi}^{+} \tilde{\chi}^{-}}$is the production cross-section for the pair $\tilde{\chi}_{1}^{+} \tilde{\chi}_{1}^{-}$from the squarks decay of Eq. (18). Using public packages for computing the SUSY production crosssections [22] and decays [23], we found that $\sigma_{\tilde{\chi}^{+} \tilde{\chi}^{-}} \simeq$ $\left(\frac{1}{3} \div \frac{1}{2}\right) \sigma_{\tilde{\chi}_{2}^{0}}$ for all the points A-D we are going to study in the next section.

Since the angle between the two leptons $\theta_{\ell^{+} \ell^{-}}$from the decays of $\tilde{\chi}_{1}^{+} \tilde{\chi}_{1}^{-}$is likely to be larger than in the case of the decay of a single $\tilde{\chi}_{2}^{0}$, the background of Eq. (19) can be reduced with respect to the signal of Eq. (17) by imposing an additional cut $\theta_{\ell^{+} \ell^{-}}>\theta_{\ell^{+} \ell^{-}}^{\min }$ [11]. With an appropriate choice of $\theta_{\ell^{+} \ell^{-}}$, the number of background events $B_{\ell^{+} \ell^{-}}^{\left(\tilde{\chi}^{+} \tilde{\chi}^{-}\right)}$should be sufficiently reduced at least in the case of same flavour leptons 11].

A second source of background events comes from the neutralino decays into taus, followed by the decay of taus into leptons:

$$
\begin{aligned}
B_{\ell^{+} \ell^{-}}^{\tau \tau} & =\sigma_{\tilde{\chi}_{2}^{0}} \times \epsilon_{\ell}^{2} \times \epsilon_{2 \tau_{\ell}} \times \epsilon_{\mathrm{cut}} \times L \times \\
& \times \operatorname{BR}\left(\tilde{\chi}_{2}^{0} \rightarrow \tilde{\chi}_{1}^{0} \tau \tau\right) \times[\operatorname{BR}(\tau \rightarrow \ell \nu \bar{\nu})]^{2},
\end{aligned}
$$

where $\operatorname{BR}(\tau \rightarrow \ell \nu \bar{\nu}) \simeq 0.17$ and $\epsilon_{2 \tau_{\ell}}$ parameterizes the acceptance of the two leptons from tau decays with respect to the acceptance of the two leptons directly produced in the decay chain of Eq. (2) . Since the former leptons should be softer than the latter, we expect $\epsilon_{2 \tau_{\ell}} \lesssim 1$. For the same reason, this source of background could be reduced by a cut on the invariant mass of the two leptons. In our case, there is an additional source of background only for the $\mu^{+} \mu^{-}$events: the flavour violating decay $\tilde{\chi}_{2}^{0} \rightarrow \tilde{\chi}_{1}^{0} \mu \tau$ followed by the decay of the tau into a muon:

$$
\begin{aligned}
B_{\mu^{+} \mu^{-}}^{\tau \mu} & =\sigma_{\tilde{\chi}_{2}^{0}} \times \epsilon_{\ell}^{2} \times \epsilon_{1 \tau_{\ell}} \times \epsilon_{\mathrm{cut}} \times L \times \\
& \times \operatorname{BR}\left(\tilde{\chi}_{2}^{0} \rightarrow \tilde{\chi}_{1}^{0} \mu \tau\right) \operatorname{BR}(\tau \rightarrow \mu \nu \bar{\nu}),
\end{aligned}
$$

where $\epsilon_{1 \tau_{\ell}}$ is the relative acceptance for a lepton coming from a tau decay.

Let us now consider the LFV neutralino decay. The total number of LFV $\tau^{+} \mu^{-}$and $\tau^{-} \mu^{+}$events is given by:

$$
\begin{aligned}
S_{\tau \mu} & =2 \times \sigma_{\tilde{\chi}_{2}^{0}} \times \epsilon_{\tau_{h}} \times \epsilon_{\ell} \times \epsilon_{\text {cut }} \times L \times \\
& \times \operatorname{BR}\left(\tilde{\chi}_{2}^{0} \rightarrow \tilde{\chi}_{1}^{0} \tau \mu\right) \operatorname{BR}(\tau \rightarrow h),
\end{aligned}
$$

where only hadronically decaying taus $\tau \rightarrow h$ have been considered (with $\operatorname{BR}(\tau \rightarrow h) \simeq 65 \%$ ) and $\epsilon_{\tau_{h}}$ is the detection efficiency for a tau jet. 
Similarly to the flavour conserving case, the SUSY background is mainly given by $\tilde{\chi}_{1}^{+} \tilde{\chi}_{1}^{-}$decays and by ditau neutralino events:

$$
\begin{aligned}
B_{\tau \mu}^{\tilde{\chi}^{+} \tilde{\chi}^{-}} & =2 \times \sigma_{\tilde{\chi}^{+}} \tilde{\chi}^{-} \times \epsilon_{\tau_{h}} \times \epsilon_{\ell} \times \epsilon_{\mathrm{cut}} \times \operatorname{BR}(\tau \rightarrow h) \times \\
& \times \operatorname{BR}\left(\tilde{\chi}_{1}^{ \pm} \rightarrow \tau^{ \pm} X\right) \operatorname{BR}\left(\tilde{\chi}_{1}^{ \pm} \rightarrow \mu^{ \pm} X\right), \\
B_{\tau \mu}^{\tau \tau} & =2 \times \sigma_{\tilde{\chi}_{2}^{0}} \times \epsilon_{\ell} \times \epsilon_{\tau_{h}} \times \epsilon_{1 \tau_{\ell}} \times \epsilon_{\mathrm{cut}} \times L \times \\
& \times \operatorname{BR}\left(\tilde{\chi}_{2}^{0} \rightarrow \tilde{\chi}_{1}^{0} \tau \tau\right) \operatorname{BR}(\tau \rightarrow h) \operatorname{BR}(\tau \rightarrow \mu \nu \bar{\nu}) .
\end{aligned}
$$

As in the flavour conserving case, the background from $\tilde{\chi}^{+} \tilde{\chi}^{-}$can be reduced with cuts on the angle between the muon and the tau-jet.

As we will see in the following, the number of background events of Eqs. (23, 24) can easily overwhelm the signal. However, an equal number of $\tau-e$ events is expected to come from the processes contributing to the $\tau-\mu$ background while, as previously mentioned, we cannot have simultaneously sizeable LFV both in the $\tau-\mu$ and in the $\tau-e$ sectors because of the $\mu \rightarrow e \gamma$ constraints. Therefore, the subtraction of the number of $\tau e$ events from the number of $\tau-\mu$ events should allow us to cancel the background, so that an excess of $\tau-\mu$ events would be a signal of LFV [5, 6]. In particular, the observation of $\tau-\mu \mathrm{LFV}$ at the LHC should be possible, as long as $\operatorname{BR}\left(\tilde{\chi}_{2}^{0} \rightarrow \tilde{\chi}_{1}^{0} \tau \mu\right) / \operatorname{BR}\left(\tilde{\chi}_{2}^{0} \rightarrow \tilde{\chi}_{1}^{0} \tau \tau\right) \gtrsim 0.1[\underline{6}]$.

\section{REPRESENTATIVE POINTS}

We consider the following representative points of the parameter space studied in section IV (for all $\tan \beta=10$, $\left.A_{0}=0\right)$ :

Point A: $m_{0}=90 \mathrm{GeV}, M_{1 / 2}=400 \mathrm{GeV}$ and $\left(\delta_{\mathrm{LL}}\right)_{32}=0.03$. This point lies in the neutralino-stau coannihilation region and reduces the $(g-2)_{\mu}$ tension below the $2-\sigma$ level. $\operatorname{BR}(\tau \rightarrow \mu \gamma)$ is predicted to be just below the present bound (see the top panel of Fig. 3).

Point B: $m_{0}=105 \mathrm{GeV}, M_{1 / 2}=500 \mathrm{GeV}$ and $\left(\delta_{\mathrm{LL}}\right)_{32}=0.03$. This point lies in the coannihilation region as shown by the top panel of Fig. 3 but gives a smaller SUSY contribution to $(g-2)_{\mu}$ and $\operatorname{BR}(\tau \rightarrow \mu \gamma)$ compared to Point A.

Point C: $m_{0}=90 \mathrm{GeV}, M_{1 / 2}=350 \mathrm{GeV}$ and $\left(\delta_{\mathrm{RR}}\right)_{32}=0.1$. This point lies in the neutralino-stau coannihilation region and reduces the $(g-2)_{\mu}$ tension below the 2- $\sigma$ level. $\operatorname{BR}(\tau \rightarrow \mu \gamma)$ is predicted to be just below the present bound (see the bottom panel of Fig. (3).

Point D: $m_{0}=150 \mathrm{GeV}, M_{1 / 2}=300 \mathrm{GeV},\left(\delta_{\mathrm{RR}}\right)_{32}=$ 0.1 . This point does not lie in the coannihilation region, but it is still in the region where the $\tilde{\chi}_{2}^{0}$ decays into $\tilde{e}_{R}$ and $\tilde{\mu}_{R}$ are kinematically allowed (while the decays into LH sleptons are forbidden). The $(g-2)_{\mu}$ tension is reduced below the 2- $\sigma$ level and $\operatorname{BR}(\tau \rightarrow \mu \gamma)$ attains experimentally visible values.

In Tab. II we present the relevant cross-sections of Eqs. (16) for the points listed above, as well as the corresponding mass splitting $\left|\Delta m_{\tilde{\ell}} / m_{\tilde{\ell}}\right|$ and the edge splitting
$\left|\Delta m_{l l} / m_{l l}\right|$. The resulting $a_{\mu}^{\mathrm{SUSY}}$ and $\operatorname{BR}(\tau \rightarrow \mu \gamma)$ are also provided.

Estimates of signals and backgrounds discussed in the previous section are given in Tab. [II] An integrate luminosity of $L=100 \mathrm{fb}^{-1}$ has been assumed. We employed the results of the previous section together with numerical computation of SUSY production crosssection [22] and decay branching fractions [23]. Following [11], we assumed the following values for the parameters: $\epsilon_{\text {cut }}=1 / 4, \epsilon_{\ell}=0.9, \epsilon_{2 \tau_{\ell}}=\epsilon_{1 \tau_{\ell}}=1, \epsilon_{\tau_{h}}=0.7$.

As a general comment, we see that the flavour conserving channels should be distinguished from the background, especially if $B_{\ell^{+} \ell^{-}}^{\tilde{\chi}^{+}} / S_{\ell^{+} \ell^{-}}$could be reduced by a cut on the angle between the leptons, $\theta_{\ell^{+} \ell^{-}}$(clearly, this would reduce the number of the signal events, but increasing the $\mathrm{S} / \mathrm{B}$ ratio). An exception is represented by point D: in this case the large background $B_{\mu^{+} \mu^{-}}^{(\tau \tau)}$ could make the measurement of the smuon mass very challenging, unless a lower cut on the di-muon invariant mass could decrease the background. For the other points, it should be possible to extract the slepton masses from the di-lepton invariant mass distributions. Of course, in order to do so, a quite large integrated luminosity (such as the $100 \mathrm{fb}^{-1}$ considered in Tab. III), i.e. some years of data taking, is probably necessary in order to collect enough statistics.

Concerning the LFV channel, we see that the detection condition $\operatorname{BR}\left(\tilde{\chi}_{2}^{0} \rightarrow \tilde{\chi}_{1}^{0} \tau \mu\right) / \operatorname{BR}\left(\tilde{\chi}_{2}^{0} \rightarrow \tilde{\chi}_{1}^{0} \tau \tau\right) \gtrsim 0.1[\underline{6}]$ is satisfied for all the points. In particular, detection of $\tilde{\chi}_{2}^{0} \rightarrow \tilde{\chi}_{1}^{0} \tau \mu$ looks very promising for Points $\mathrm{C}$ and $\mathrm{D}$, due to the relatively low background and the large ratio $\operatorname{BR}\left(\tilde{\chi}_{2}^{0} \rightarrow \tilde{\chi}_{1}^{0} \tau \mu\right) / \operatorname{BR}\left(\tilde{\chi}_{2}^{0} \rightarrow \tilde{\chi}_{1}^{0} \tau \tau\right) \simeq 0.3$.

Let us now go in detail through the results presented in Tabs. IIII in order to show, in particular, the possible interplay among low-energy and collider searches for LFV.

Let us first consider Point A. We see that the per-cent mass splitting between $\tilde{e}_{L}$ and $\tilde{\mu}_{L}$ induced by $\left(\delta_{\mathrm{LL}}\right)_{32}$ results in a large $10 \%$ splitting of the $e-e$ and $\mu-\mu$ edges. Moreover, we checked that an intermediate $\tilde{e}_{R}\left(\tilde{\mu}_{R}\right)$ gives a contribution to $\Gamma\left(\tilde{\chi}_{2}^{0} \rightarrow \tilde{\chi}_{1}^{0} e^{+} e^{-}\right)\left(\Gamma\left(\tilde{\chi}_{2}^{0} \rightarrow \tilde{\chi}_{1}^{0} \mu^{+} \mu^{-}\right)\right)$ which is around $20 \%$. As a consequence, the kinematical end points corresponding to $\tilde{e}_{L}$ and $\tilde{\mu}_{L}$ should not be hidden by a large number of events mediated by righthanded sleptons. Thus, we can expect $\Delta m_{\tilde{\ell}} / m_{\tilde{\ell}}$ to be measured at the LHC. $\operatorname{BR}(\tau \rightarrow \mu \gamma)$ is predicted in the reach of the future experiments. Furthermore, the LFV neutralino decay has also a good probability to be detected (we find $\operatorname{BR}\left(\tilde{\chi}_{2}^{0} \rightarrow \tilde{\chi}_{1}^{0} \tau^{ \pm} \mu^{\mp}\right) \simeq 3 \%$, which corresponds to $\left.\operatorname{BR}\left(\tilde{\chi}_{2}^{0} \rightarrow \tilde{\chi}_{1}^{0} \tau \mu\right) / \operatorname{BR}\left(\tilde{\chi}_{2}^{0} \rightarrow \tilde{\chi}_{1}^{0} \tau \tau\right) \simeq 0.1\right)$. We can conclude that Point A provides an example in which all the LFV observables we discussed so far could provide a positive signal. Hence, in this case, the combined analysis of all the above observables would represent a powerful tool in the attempt to reconstruct the flavour structure of the slepton sector.

Point B represents a case where the LHC could provide positive signals of LFV better than the direct searches for 


\begin{tabular}{|c|c|c|c|c|c|c|c|c|}
\hline & $\sigma_{\mathrm{SUSY}}$ & $\sigma_{e e}$ & $\sigma_{\mu \mu}$ & $\sigma_{\tau \mu}$ & $\left|\Delta m_{\tilde{\ell}} / m_{\tilde{\ell}}\right|$ & $\left|\Delta m_{l l} / m_{l l}\right|$ & $a_{\mu}^{\mathrm{SUSY}}$ & $\mathrm{BR}(\tau \rightarrow \mu \gamma)$ \\
\hline \hline Point A & $5.2 \mathrm{pb}$ & $63 \mathrm{fb}$ & $43 \mathrm{fb}$ & $24 \mathrm{fb}$ & $1.1 \%$ & $10 \%$ & $1.2 \times 10^{-9}$ & $1.7 \times 10^{-8}$ \\
Point B & $1.8 \mathrm{pb}$ & $32 \mathrm{fb}$ & $18 \mathrm{fb}$ & $15 \mathrm{fb}$ & $1.3 \%$ & $7.6 \%$ & $8.0 \times 10^{-10}$ & $7.3 \times 10^{-9}$ \\
Point C & $9.7 \mathrm{pb}$ & $62 \mathrm{fb}$ & $49 \mathrm{fb}$ & $110 \mathrm{fb}$ & $2.7 \%$ & $4.9 \%$ & $1.5 \times 10^{-9}$ & $2.4 \times 10^{-8}$ \\
Point D & $18.2 \mathrm{pb}$ & $169 \mathrm{fb}$ & $91 \mathrm{fb}$ & $536 \mathrm{fb}$ & $3.0 \%$ & $6.2 \%$ & $1.6 \times 10^{-9}$ & $1.3 \times 10^{-8}$ \\
\hline
\end{tabular}

TABLE II: Predictions for low and high energy observables for the Points A,B,C,D discussed in the text.

\begin{tabular}{|c|c|c|c|c|c|c|c|c|c|c|c|}
\hline & $S_{\mu^{+} \mu^{-}}$ & $\begin{array}{c}B_{\mu^{+} \mu^{-}}^{\left(\tilde{\chi}^{+} \tilde{\chi}^{-}\right)} \\
\end{array}$ & $B_{\mu^{+} \mu^{-}}^{(\tau \tau)}$ & $B_{\mu^{+} \mu^{-}}^{(\tau \mu)}$ & $S_{e^{+e^{-}}}$ & $\begin{array}{c}B_{e^{+} e^{-}}^{\left(\tilde{\chi}^{+} \tilde{\chi}^{-}\right)} \\
\end{array}$ & $\begin{array}{c}B_{e^{+} e^{-}}^{(\tau \tau)} \\
\end{array}$ & $S_{\tau \mu}$ & $B_{\tau \mu}^{\left(\tilde{\chi}^{+} \tilde{\chi}^{-}\right)}$ & $B_{\tau \mu}^{(\tau \tau)}$ & $\frac{\operatorname{BR}(\tau \mu)}{\operatorname{BR}(\tau \tau)}$ \\
\hline it $A$ & 850 & $0.65 S_{\mu^{+} \mu^{-}}$ & $0.12 S_{\mu^{+} \mu^{-}}$ & $0.09 S_{\mu^{+} \mu^{-}}$ & 1275 & $0.44 S_{e^{+e^{-}}}$ & $0.09 S_{e^{+} e^{-}}$ & 490 & $1.15 S_{\tau \mu}$ & $1.3 S_{\tau \mu}$ & 0.12 \\
\hline Point B & 364 & $0.64 S_{\mu^{+} \mu^{-}}$ & $0.07 S_{\mu^{+} \mu^{-}}$ & $0.14 S_{\mu^{+} \mu^{-}}$ & 648 & $0.35 S_{e^{+} e^{-}}$ & $0.04 S_{e^{+e^{-}}}$ & 307 & $0.82 S_{\tau \mu}$ & $0.53 S_{\tau \mu}$ & 0.32 \\
\hline Point C & 992 & $0.48 S_{\mu^{+} \mu^{-}}$ & $0.19 S_{\mu^{+} \mu^{-}}$ & $0.38 S_{\mu^{+} \mu^{-}}$ & 1255 & $0.38 S_{e^{+} e^{-}}$ & $0.15 S_{e^{+} e^{-}}$ & 1126 & $0.21 S_{\tau \mu}$ & $0.5 S_{\tau \mu}$ & 0.34 \\
\hline Point D & 1842 & $0.16 S_{\mu^{+} \mu^{-}}$ & $0.45 S_{\mu^{+} \mu^{-}}$ & $1.02 S_{\mu^{+} \mu^{-}}$ & 3822 & $0.09 S_{e^{+e^{-}}}$ & $0.24 S_{e^{+} e^{-}}$ & 10974 & $0.03 S_{\tau \mu}$ & $0.44 S_{\tau \mu}$ & 0.38 \\
\hline
\end{tabular}

TABLE III: Expected number of signal and background events for the relevant flavour conserving and violating channels. The estimate has been done taking for the integrated luminosity $L=100 \mathrm{fb}^{-1}$.

$\tau \rightarrow \mu \gamma$, which is predicted to be beyond the reach of a SuperB factory at KEK [17] but still within the reach of a Super Flavour Factory [21]. The measurement of the slepton mass splitting would for sure require a larger integrated luminosity than what is required by Point A. Still, the prospects at the LHC are reasonably favorable, given the low contribution of the $\mathrm{RH}$ sleptons to the $\tilde{\chi}_{2}^{0}$ decay widths (at around the $10 \%$ level) and the large LFV rate $\operatorname{BR}\left(\tilde{\chi}_{2}^{0} \rightarrow \tilde{\chi}_{1}^{0} \tau^{ \pm} \mu^{\mp}\right) \simeq 5 \%$.

Passing to Point C, we like to emphasize that, in spite of similar predictions for the production cross sections to the Point A, we find that the measurement of the mass splitting between $\tilde{e}_{R}$ and $\tilde{\mu}_{R}$ is more challenging. The reason is that we are in a kinematic region where the decays of $\tilde{\chi}_{2}^{0}$ into LH sleptons are open. As a consequence, since $\tilde{\chi}_{2}^{0}$ is mostly Wino, the contribution to the decay widths $\Gamma\left(\tilde{\chi}_{2}^{0} \rightarrow \tilde{\chi}_{1}^{0} e^{+} e^{-}\right)$and $\Gamma\left(\tilde{\chi}_{2}^{0} \rightarrow \tilde{\chi}_{1}^{0} \mu^{+} \mu^{-}\right)$ from intermediate LH sleptons is almost twice the contribution from $\tilde{e}_{R}$ and $\tilde{\mu}_{R}$. Even though the production cross sections that are effective for the measurement of the mass splitting for right-handed sleptons are much smaller than the total production cross sections (that are dominated by the contributions of LH sleptons), we notice that the kinematical end points for the invariant mass distributions of right-handed sleptons appear at higher values compared to those of left-handed sleptons; as a result, the measurements of the kinematic edges associated with the RH sleptons should not be affected by a large background. Therefore, $\Delta m_{\tilde{\ell}} / m_{\tilde{\ell}}$ could be still measured provided enough statistics. Moreover, Point $\mathrm{C}$ gives a very large rate to the LFV $\tilde{\chi}_{2}^{0}$ decay (being $\operatorname{BR}\left(\tilde{\chi}_{2}^{0} \rightarrow \tilde{\chi}_{1}^{0} \tau^{ \pm} \mu^{\mp}\right) \simeq 7 \%$ ) and a prediction for $\operatorname{BR}(\tau \rightarrow \mu \gamma)$ in the reach of the future experiments.

Finally, Point D, being in a region where the $\tilde{\chi}_{2}^{0}$ decays into LH sleptons are not allowed, could give good prospects for the measurement of the $\tilde{e}_{R}-\tilde{\mu}_{R}$ mass splitting, only if the background to the $\mu \mu$ events coming from the LFV neutralino decay $\left(B_{\mu^{+} \mu^{-}}^{(\tau \mu)}\right)$ could reduced with a lower cut on the di-muon invariant mass. Another caveat is that around the $30 \%$ of the "pure" $\mu-\mu$ events are mediated by $\tilde{\tau}_{1}$, so that the di-muon distribution will exhibit two edges, one corresponding to the kinematical end-point of $\tilde{\mu}_{R}$, the other one corresponding to $\tilde{\tau}_{1}$. On the other hand, the huge cross-section of $\tilde{\chi}_{2}^{0} \rightarrow \tilde{\chi}_{1}^{0} \tau^{ \pm} \mu^{\mp}$ (up to a factor of 3-6 larger than the cross-sections of the flavour conserving decays) and the relatively low background make the LFV $\tilde{\chi}_{2}^{0}$ decay the most sensitive LFV observable.

\section{CONCLUSIONS}

In this paper, we have studied the sensitivity of the slepton mass splittings of the first two generations to LFV effects.

In particular, we have considered minimal SUGRA scenarios, predicting highly degenerate first two slepton generations. Hence, any experimental evidence for a selectron/smuon mass splitting $\Delta m_{\tilde{\ell}} / m_{\tilde{\ell}}$, say above the percent level, would signal either a different mechanism for the SUSY breaking or non minimal realizations of SUGRA models. In the latter case, the presence of LFV interactions might explain the potential evidence of a significant $\Delta m_{\tilde{\ell}} / m_{\tilde{\ell}}$, as throughly discussed in this paper.

Interestingly enough, we have found that sizable values for $\Delta m_{\tilde{\ell}} / m_{\tilde{\ell}}$ can be generated only through flavour mixings between the second and third slepton families, a scenario that naturally arises and is well motivated by the large mixing angle observed in atmospheric neutrino oscillation experiments.

In contrast, flavour mixings between the second and first slepton families are highly constrained by the limit on $\operatorname{BR}(\mu \rightarrow \mathrm{e} \gamma)$ and they can hardly account for such a mass splitting [3]. 
We have shown that slepton mass splittings may be competitive with low energy processes to probe lepton flavour violation in large regions of the SUSY parameter space.

Moreover, we have exploited the NP sensitivity of the high energy LFV process $\tilde{\chi}_{2}^{0} \rightarrow \tilde{\chi}_{1}^{0} \tau^{ \pm} \mu^{\mp}$ assuming an integrated luminosity $L=100 \mathrm{fb}^{-1}$. We have found that, whenever the relevant background is kept under control by means of appropriate kinematical cuts, $\tilde{\chi}_{2}^{0} \rightarrow \tilde{\chi}_{1}^{0} \tau^{ \pm} \mu^{\mp}$ can be experimentally visible in sizable regions of the parameter space where, at the same time:

(i) $\operatorname{BR}(\tau \rightarrow \mu \gamma)$ can be below the current experimental bound and even beyond the reach of a SuperB factory at $\operatorname{KEK}\left(\operatorname{BR}(\tau \rightarrow \mu \gamma)>10^{-8}\right)$;

(ii) $a_{\mu}^{\text {SUSY }} \gtrsim 1 \times 10^{-9}$ providing an explanation for the $(g-2)_{\mu}$ anomaly, at least at the $2-\sigma$ level;

(iii) the WMAP relic density constraint can be fulfilled by an effective neutralino-stau coannihilation;

(iv) the LFV induced mass splitting between selectron and smuons can be measured at the LHC.

Hence, $\tilde{\chi}_{2}^{0} \rightarrow \tilde{\chi}_{1}^{0} \ell_{i} \ell_{j}$ and $\tau \rightarrow \mu \gamma$ might be complementary probes of LFV in SUSY with $\tilde{\chi}_{2}^{0} \rightarrow \tilde{\chi}_{1}^{0} \tau^{ \pm} \mu^{\mp}$ being even more powerful than $\tau \rightarrow \mu \gamma$ for a heavy SUSY spectrum.

In conclusion, the present study represents another proof of the synergy and interplay existing between the LHC, i.e. the high-energy frontier, and high-precision low-energy experiments, i.e. the high-intensity frontier.

Acknowledgments: We thank M. Nagai for useful discussions. This work has been supported in part by the Cluster of Excellence "Origin and Structure of the Universe" and by the German Bundesministerium für Bildung und Forschung under contract 05H09WOE.
[1] F. E. Paige, arXiv:hep-ph/9609373 I. Hinchliffe, F. E. Paige, M. D. Shapiro, J. Soderqvist and W. Yao, Phys. Rev. D 55 (1997) 5520 arXiv:hep-ph/9610544; H. Bachacou, I. Hinchliffe and F. E. Paige, Phys. Rev. D 62 (2000) 015009 arXiv:hep-ph/9907518.

[2] W. W. Armstrong et al. [ATLAS Collaboration], CERNLHCC-94-43;G. L. Bayatian et al. [CMS Collaboration], J. Phys. G 34, 995 (2007).

[3] J. Hisano, R. Kitano and M. M. Nojiri, Phys. Rev. D 65 (2002) 116002 arXiv:hep-ph/0202129; J. Hisano, M. M. Nojiri and W. Sreethawong, JHEP 0906 (2009) 044 arXiv:0812.4496 [hep-ph]].

[4] N. Arkani-Hamed, H. C. Cheng, J. L. Feng and L. J. Hall, Phys. Rev. Lett. 77 (1996) 1937 arXiv:hep-ph/9603431; N. Arkani-Hamed, J. L. Feng, L. J. Hall and H. C. Cheng, Nucl. Phys. B 505 (1997) 3 arXiv:hep-ph/9704205.

[5] I. Hinchliffe and F. E. Paige, Phys. Rev. D 63 (2001) 115006 arXiv:hep-ph/0010086.

[6] D. F. Carvalho, J. R. Ellis, M. E. Gomez, S. Lola and J. C. Romao, Phys. Lett. B 618 (2005) 162 arXiv:hep-ph/0206148; E. Carquin, J. Ellis, M. E. Gomez, S. Lola and J. Rodriguez-Quintero, JHEP 0905 (2009) 026 arXiv:0812.4243 [hep-ph]].

[7] F. del Aguila and L. Ametller, Phys. Lett. B 261 (1991) 326; H. Baer, C. h. Chen, F. Paige and X. Tata, Phys. Rev. D 49 (1994) 3283 arXiv:hep-ph/9311248; Yu. M. Andreev, S. I. Bityukov and N. V. Krasnikov, Phys. Atom. Nucl. 68 (2005) 340 [Yad. Fiz. 68 (2005) 366] arXiv:hep-ph/0402229.

[8] B. C. Allanach, J. P. Conlon and C. G. Lester, Phys. Rev. D 77, 076006 (2008) arXiv:0801.3666 [hep-ph]].

[9] Y. Nir and N. Seiberg, Phys. Lett. B 309 (1993) 337 arXiv:hep-ph/9304307; Y. Nir and G. Raz, Phys. Rev. D 66 (2002) 035007 arXiv:hep-ph/0206064.

[10] J. L. Feng, C. G. Lester, Y. Nir and Y. Shadmi, Phys. Rev. D 77, 076002 (2008) arXiv:0712.0674 [hep-ph]]; J. L. Feng, S. T. French, C. G. Lester, Y. Nir and Y. Shadmi, Phys. Rev. D 80 (2009) 114004 arXiv:0906.4215 [hep-ph]]; J. L. Feng et al.,
arXiv:0910.1618 [hep-ph]; Y. Nomura, M. Papucci and D. Stolarski, Phys. Rev. D 77 (2008) 075006 arXiv:0712.2074 [hep-ph]].

[11] K. Agashe and M. Graesser, Phys. Rev. D 61, 075008 (2000) arXiv:hep-ph/9904422.

[12] For further studies about neutralino LFV decay modes, please see: J. Hisano, M. M. Nojiri, Y. Shimizu and M. Tanaka, Phys. Rev. D 60 (1999) 055008 arXiv:hep-ph/9808410; A. Bartl, K. Hidaka, K. Hohenwarter-Sodek, T. Kernreiter, W. Majerotto and W. Porod, Eur. Phys. J. C 46 (2006) 783 arXiv:hep-ph/0510074.

[13] P. Paradisi, JHEP 0510, $006 \quad$ (2005) arXiv:hep-ph/0505046.

[14] A. Ibarra and C. Simonetto, JHEP 0804 (2008) 102 arXiv:0802.3858 [hep-ph]].

[15] A. Djouadi, Y. Mambrini and M. Muhlleitner, Eur. Phys. J. C 20 (2001) 563 arXiv:hep-ph/0104115; T. Goto, K. Kawagoe and M. M. Nojiri, Phys. Rev. D 70 (2004) 075016 [Erratum-ibid. D 71 (2005) 059902] arXiv:hep-ph/0406317.

[16] Y. Miyazaki et al. [Belle Collaboration], Phys. Lett. B 660 (2008) 154 arXiv:0711.2189 [hep-ex]]; B. Aubert et al. [BABAR Collaboration], arXiv:0908.2381 [hep-ex].

[17] A. G. Akeroyd et al. [SuperKEKB Physics Working Group], arXiv:hep-ex/0406071

[18] A. A. Alves et al. [LHCb Collaboration], JINST 3 (2008) S08005.

[19] J. Hisano, T. Moroi, K. Tobe and M. Yamaguchi, Phys. Rev. D 53 (1996) 2442 arXiv:hep-ph/9510309.

[20] W. Altmannshofer, A. J. Buras, S. Gori, P. Paradisi and D. M. Straub, Nucl. Phys. B 830 (2010) 17 arXiv:0909.1333 [hep-ph]].

[21] M. Bona et al., arXiv:0709.0451 [hep-ex].

$[22]$ W. Beenakker, R. Hopker and M. Spira, arXiv:hep-ph/9611232.

[23] A. Djouadi, M. M. Muhlleitner and M. Spira, Acta Phys. Polon. B 38 (2007) 635 arXiv:hep-ph/0609292. 